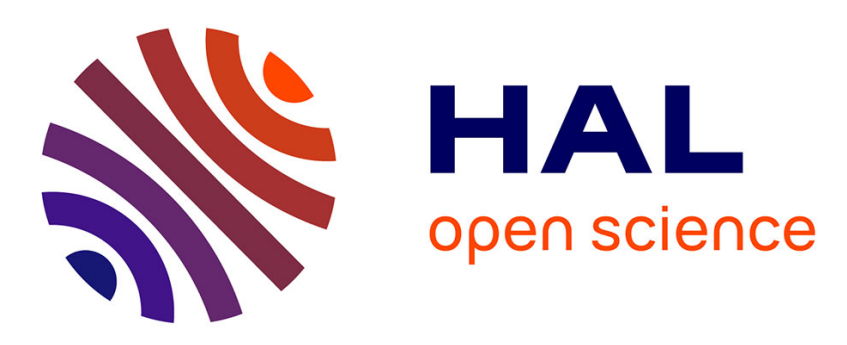

\title{
Role of Roots of Orthogonal Polynomials in the Dynamic Response of Stochastic Systems
}

Eric Jacquelin, Olivier Dessombz, Sondipon Adhikari, Michael I Friswell, Jean-Jacques Sinou

\section{To cite this version:}

Eric Jacquelin, Olivier Dessombz, Sondipon Adhikari, Michael I Friswell, Jean-Jacques Sinou. Role of Roots of Orthogonal Polynomials in the Dynamic Response of Stochastic Systems. Journal of Engineering Mechanics - ASCE, 2016, 142 (8), 10.1061/(ASCE)EM.1943-7889.0001102 . hal-01356465v2

\section{HAL Id: hal-01356465 \\ https://hal.science/hal-01356465v2}

Submitted on 31 May 2017

HAL is a multi-disciplinary open access archive for the deposit and dissemination of scientific research documents, whether they are published or not. The documents may come from teaching and research institutions in France or abroad, or from public or private research centers.
L'archive ouverte pluridisciplinaire HAL, est destinée au dépôt et à la diffusion de documents scientifiques de niveau recherche, publiés ou non, émanant des établissements d'enseignement et de recherche français ou étrangers, des laboratoires publics ou privés. 


\title{
The role of roots of orthogonal polynomials in the dynamic response of stochastic systems
}

\author{
E. Jacquelin ${ }^{\mathrm{a}, \mathrm{b}, \mathrm{c}}$, O. Dessombz ${ }^{\mathrm{d}}$, S. Adhikari ${ }^{\mathrm{e}}$, M.I. Friswelle ${ }^{\mathrm{e}}$,J.-J. Sinou ${ }^{\mathrm{d}, \mathrm{f}}$ \\ ${ }^{a}$ Université de Lyon, F-69622, Lyon, France. \\ ${ }^{b}$ Université Claude Bernard Lyon 1, Villeurbanne. \\ 'IFSTTAR, UMR-T9406, LBMC Laboratoire de Biomécanique et Mécanique des chocs, \\ F69675, Bron. \\ ${ }^{d}$ École Centrale de Lyon, LTDS, UMR CNRS 5513, F-69134, Écully - France. \\ ${ }^{e}$ College of Engineering, Swansea University, Swansea SA2 8PP, UK. \\ ${ }^{f}$ Institut Universitaire de France, 75005 Paris, France.
}

\begin{abstract}
This note investigates the fundamental nature of the polynomial chaos (PC) response of dynamic systems with uncertain parameters in the frequencydomain. The eigenfrequencies of the extended matrix arising from a PC formulation govern the convergence of the dynamic response. It is shown that, in the particular case of uncertainties and with Hermite and Legendre polynomials, the PC-eigenfrequencies are related to the roots of the underlying polynomials, which belongs to the polynomial chaos set used to derive the polynomial chaos expansion. When Legendre polynomials are used, the PC-eigenfrequencies remain in a bounded interval close to the deterministic eigenfrequencies, as they are related to the roots of a Legendre polynomial. The higher the PC order, the higher the density of the PC-eigenfrequencies
\end{abstract}

\footnotetext{
${ }^{*}$ Corresponding author: eric.jacquelin@univ-lyon1.fr, Tel: +33478931671

Email addresses: S.Adhikari@swansea.ac.uk (S. Adhikari), M.I.Friswell@swansea.ac.uk (M.I. Friswell), Jean-Jacques.Sinou@ec-lyon.fr (J.-J. Sinou)
}

Preprint submitted to Journal of Engineering Mechanics - Accepted, February 15, 2016 
close to the bounds of the interval, and this tends to smooth the frequency response quickly. In contrast, when Hermite polynomials are used, the PCeigenfrequencies spread from the deterministic eigenfrequencies (the highest roots of the Hermite polynomials tend to infinity when the order tends to infinity). Consequently, when the PC number increases, the smoothing effect becomes inefficient.

Keywords: Random dynamical systems; polynomial chaos expansion; steady-state response; convergence; roots of orthogonal polynomials

\section{Introduction}

The dynamic analysis of multiple-degree-of-freedom linear systems with parametric uncertainties has received significant attention over the past decade. There are three main routes to solve this problem, namely (a) via a random modal analysis, (b) an integration of the coupled random equations of motion in the time domain, or (c) by directly solving the (complex) equations of motion in the frequency domain. For all three approaches, several reduced computational methods are available which avoid the use of expensive direct Monte Carlo simulations $[9,10]$. Falsone and co-workers have proposed a modified perturbation method [4] and the so-called APDM-based method [12] to efficiently solve the uncertain static problem. The modified perturbation method has also been extended to uncertain dynamical systems [3]. Polynomial chaos expansion (PCE) [6] has been used extensively for all three approaches in the context of a dynamic system $[1,5,14]$. This paper focusses on the third approach, that is the frequency domain solution of the dynamic response using PCE. 
From an engineering point of view, the response near the resonance frequency is of paramount importance. The nature of the PC response around the deterministic resonance frequency of a random dynamic system can be significantly different from any other points in the frequency axis. It was shown that inadequate polynomial orders lead to spurious peaks in the dynamic response around the deterministic resonance frequency. This was attributed to eigenfrequencies of the augmented system matrices that one obtained in conjunction with the PC expansion coefficients.

Up until now it is normally considered that the computational cost and accuracy of a PC solution for a given problem depend mainly on the order of the polynomials. This in turn determines the number of terms in the PC expansion depending on the number of random variables. The nature of the polynomials on their own is not a contributing factor as long as the correct polynomials are chosen based on the underlying probability density function. However, for structural dynamic problems this may not always be the case. This note aims to explain why the PCE convergence is faster with Legendre polynomials compared to Hermite polynomials when the stiffness matrix is random. The convergence rate is shown to depend on the nature of distribution of roots of such polynomials.

\section{Response of a random dynamical system}

Consider an $n$ degrees of freedom (dof) dynamical system described by its mass, damping and stiffness matrices, $\mathbf{M}, \mathbf{D}$ and $\mathbf{K}$. The forces acting on this system are described by $\mathbf{F}(t)$, and $\mathbf{x}(t)$ denotes the response vector, 
which is the solution of

$$
\mathbf{M} \ddot{\mathbf{x}}(t)+\mathbf{D} \dot{\mathbf{x}}(t)+\mathbf{K} \mathbf{x}(t)=\mathbf{F}(t)
$$

The mass, damping and stiffness matrices are assumed to be uncertain and given by

$$
\begin{aligned}
\mathbf{M}=\mathbf{M}(\Xi) & =\sum_{i=0}^{r} \xi_{i} \mathbf{M}_{i} \\
\mathbf{D}=\mathbf{D}(\Xi) & =\sum_{i=0}^{r} \xi_{i} \mathbf{D}_{i} \\
\mathbf{K}=\mathbf{K}(\Xi) & =\sum_{i=0}^{r} \xi_{i} \mathbf{K}_{i}
\end{aligned}
$$

where $\Xi=\left(\xi_{1}, \ldots, \xi_{r}\right)$ and $\xi_{i>0}$ is a zero-mean random variable; $\xi_{0}=1$ is not an uncertain variable.

The solution of Eq. (1) is random and may be expanded in terms of the $\mathrm{PC}$ basis $\left\{\Psi_{j}(\Xi): j \in \mathbb{N}\right\}[6]$ as

$$
\mathbf{x}(t, \Xi)=\sum_{j=0}^{\infty} \mathbf{Y}_{j}(t) \Psi_{j}(\Xi)
$$

The elements of the PC basis are obtained from an orthogonal polynomial set $\left\{P_{j}(\xi): j \in \mathbb{N}\right\}$, where $j$ is the order of $P_{j}(\xi)$. Thus

$$
\Psi_{J}(\Xi)=\prod_{i=1}^{r} P_{J_{i}}\left(\xi_{i}\right)
$$

where $\sum_{i=1}^{r} J_{i}$ is the order of $\Psi_{J}$. In the following $P_{j}$ is the Hermite (resp. Legendre) polynomial, $H_{j}$ (resp. $L_{j}$ ), when $\xi_{i}$ is a normally (resp. uniform) distributed random variable. This choice is not obvious [11] but it is optimal since Hermite (resp. Legendre) polynomials are orthogonal with respect to 
the inner product corresponding to the probability density function for the normal (resp. uniform) distribution [2].

For the numerical study, Eq. (5) can be truncated to a finite number of terms, $P+1$, which is given by $(m+r) ! /(m ! r !)$ where $m$ is the chaos order. Truncating the infinite expansion, gives the approximation of $\mathbf{x}(t, \Xi)$ as

$$
\mathbf{x}^{P}(t, \Xi)=\sum_{j=0}^{P} \mathbf{Y}_{j}(t) \Psi_{j}(\Xi)
$$

In the following, the exponent $P$ is dropped for the sake of simplicity.

It is easy to show that the components of the PC expansion satisfy $[7,8]$

$$
\widetilde{\mathbf{M}} \ddot{\mathbf{Y}}(t)+\widetilde{\mathbf{D}} \dot{\mathbf{Y}}(t)+\widetilde{\mathbf{K}} \mathbf{Y}(t)=\widetilde{\mathbf{F}}(t)
$$

with

$$
\begin{aligned}
& \mathbf{A}_{k} \in \mathbb{R}^{(P+1) \times(P+1)}, \quad \text { with }\left[A_{k}\right]_{I J}=<k, I, J> \\
& \widetilde{\mathbf{M}}=\sum_{k=0}^{r} \mathbf{A}_{k} \otimes \mathbf{M}_{k} \in \mathbb{R}^{2(P+1) \times 2(P+1)} \\
& \widetilde{\mathbf{D}}=\sum_{k=0}^{r} \mathbf{A}_{k} \otimes \mathbf{D}_{k} \in \mathbb{R}^{2(P+1) \times 2(P+1)} \\
& \widetilde{\mathbf{K}}=\sum_{k=0}^{r} \mathbf{A}_{k} \otimes \mathbf{K}_{k} \in \mathbb{R}^{2(P+1) \times 2(P+1)} \\
& \mathbf{Y}=\left[\begin{array}{llll}
\mathbf{Y}_{0}^{T} & \mathbf{Y}_{1}^{T} & \cdots & \mathbf{Y}_{P}^{T}
\end{array}\right]^{T} \in \mathbb{R}^{2(P+1)} \\
& \widetilde{\mathbf{F}}(t)=\left[\begin{array}{llllll}
\mathbf{F}^{T}(t) & 0 & 0 & \cdots & 0
\end{array}\right]^{T} \in \mathbb{R}^{2(P+1)}
\end{aligned}
$$

where $\otimes$ denotes the Kronecker product and $(\bullet)^{T}$ denotes the transpose of $(\bullet)$.

Hence, the PC-components are the solution of an $n(P+1)$ dof dynamical system that will be referred to as the PC-system. Thus the PCE has 


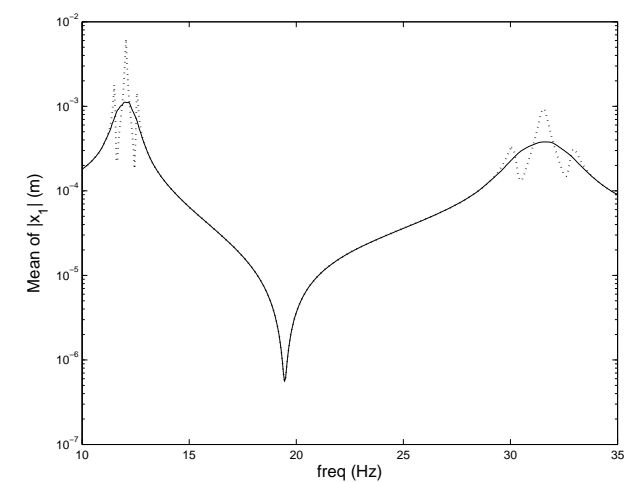

(a)

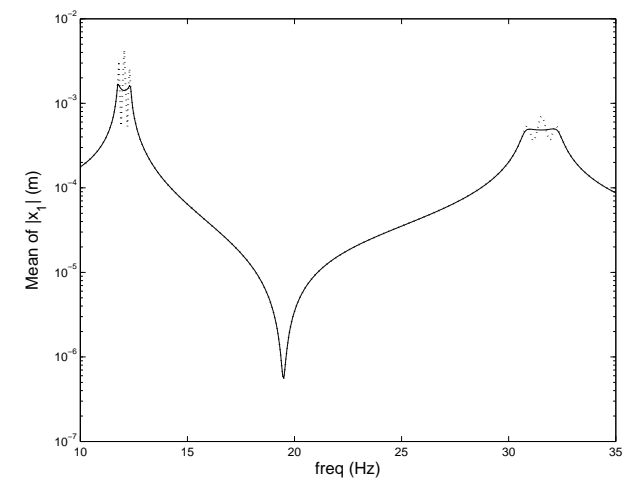

(b)

Figure 1: Mean response with MCS (solid line) and PCE of order 2 (dotted line): (a) Hermite (b) Legendre

transformed the study of an uncertain dynamical system into the study of a deterministic dynamical system of larger order. The PC-system has resonant frequencies that will be referred to as PC-resonances. As a consequence the moments of the steady-state response to a harmonic force derived through PCE show peaks related to these PC-resonances. This is highlighted by Figs. 1 and 2, which show peaks around the deterministic eigenfrequencies with both Hermite (Figs. 1(a) and 2(a)) and Legendre (Figs. 1(b) and 2(b)) polynomials of order 2. This figure is related to the example described in the next section.

This result had been already derived for a dynamical system for Hermite polynomials $[7,8,13]$. Hence, the existence of such PC-resonances has a strong influence on the convergence around the deterministic eigenfrequencies. However, Figs. 3 and 4 show that the convergence is almost reached for a PC-order equal to 10 when Legendre polynomials are used (see Figs. 3(b) and $4(\mathrm{~b})$ ) whereas the results are far from converged with Hermite polyno- 


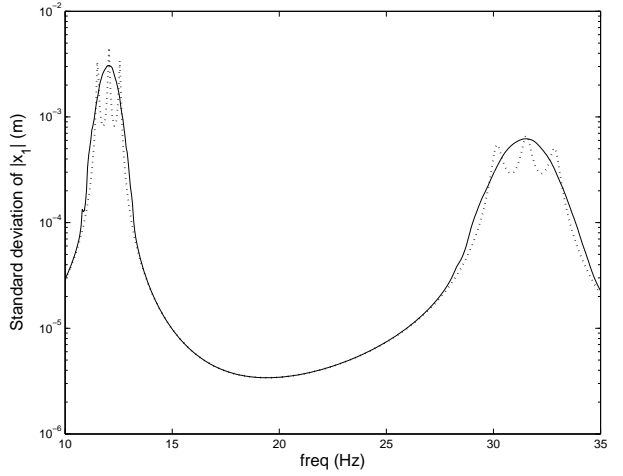

(a)

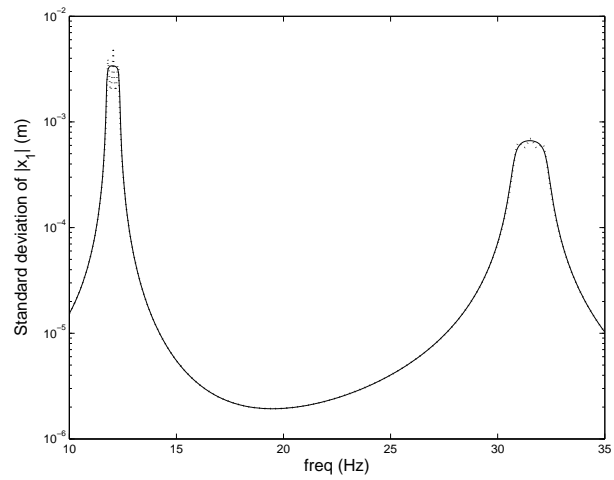

(b)

Figure 2: Standard deviation of the response with MCS (solid line) and PCE of order 2 (dotted line): (a) Hermite (b) Legendre

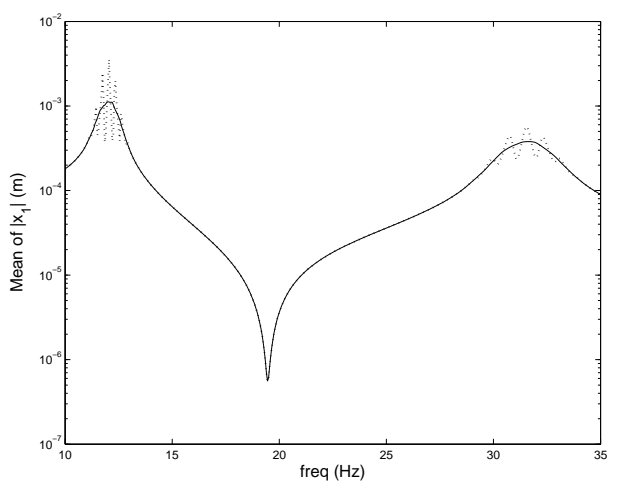

(a)

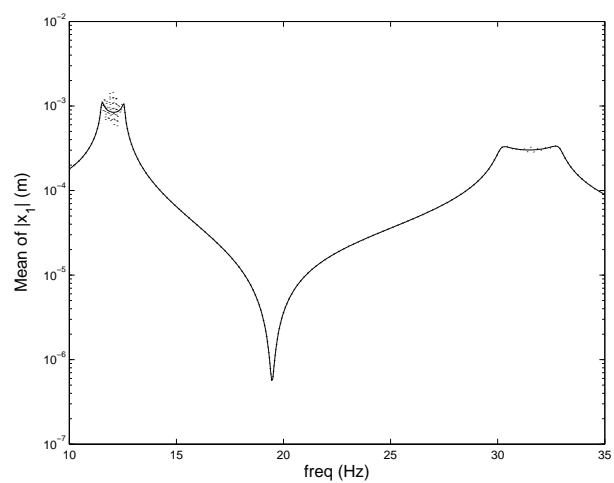

(b)

Figure 3: Mean response with MCS (solid line) and PCE of order 10 (dotted line): (a) Hermite (b) Legendre 


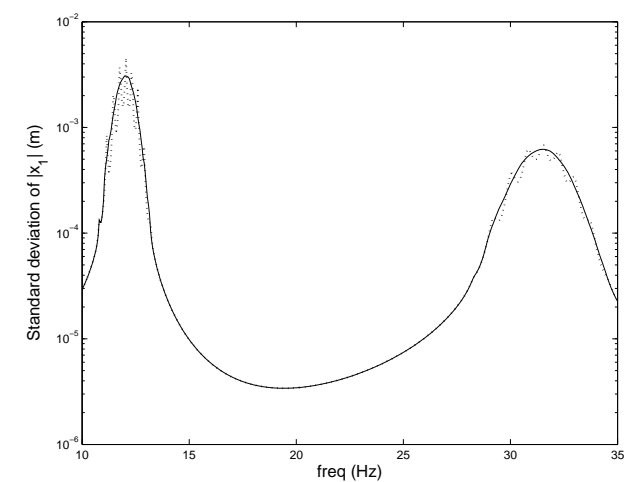

(a)

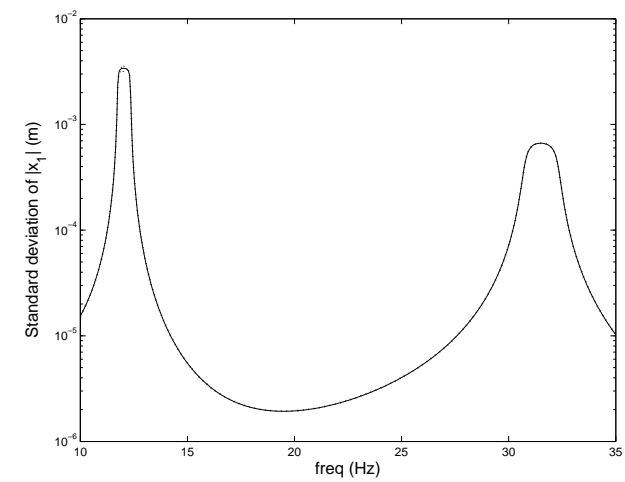

(b)

Figure 4: Standard deviation of the response with MCS (solid line) and PCE of order 10 (dotted line): (a) Hermite (b) Legendre

mials (see Figs. 3(a) and 4(a)).

\section{Uncertain stiffness matrix}

This section highlights some features related to the PC-eigenfrequencies. To enable the calculation of closed-form exact results, the mass matrix is deterministic and the uncertain stiffness matrix is assumed to be given by

$$
\mathbf{K}=\overline{\mathbf{K}}\left(1+\delta_{K} \xi\right)
$$

where $\xi$ is a standard normal or a uniform random variable and $\overline{\mathbf{K}}$ is a deterministic matrix, which represents the mean stiffness matrix. The covariance matrix of $\mathbf{K}$ is controlled by parameter $\delta_{K}$ and the deterministic dynamical system corresponds to $\delta_{K}=0$. Comparing to Eq. (4) gives $\mathbf{K}_{0}=\overline{\mathbf{K}}$ and $\mathbf{K}_{1}=\delta_{K} \overline{\mathbf{K}}$.

The appendix gives an extension proposed for uncertain mass and stiffness matrices. 


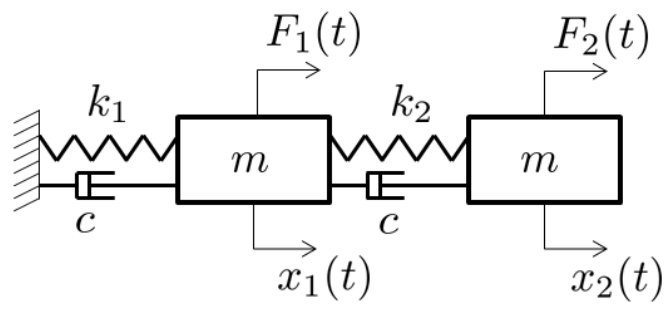

Figure 5: A two degree-of-freedom system with uncertain stiffnesses

\begin{tabular}{cccccc}
$\bar{k}\left(\mathrm{Nm}^{-1}\right)$ & $m(\mathrm{~kg})$ & $c\left(\mathrm{Nm}^{-1} \mathrm{~s}^{-1}\right)$ & $\sigma_{q}(\%)$ & $F_{01}(\mathrm{~N})$ & $F_{02}(\mathrm{~N})$ \\
\hline 15000 & 1 & 1 & 5 & 1 & 0
\end{tabular}

Table 1: System characteristics

\subsection{Description of the system}

The numerical results (see Figs. 1 and 3) are given for the example shown in Fig. 5, which represents a 2-dof dynamical system with one uncertain parameter, stiffness $k$, where

$$
k=k_{1}=k_{2}=\bar{k}\left(1+\delta_{K} \xi\right)
$$

The force vector is assumed to be harmonic, i.e. $\mathbf{F}(t)=\mathbf{F}_{\mathbf{0}} e^{\mathrm{i} \omega t}$, and the steady-state response of the dynamical system is then $\mathbf{x}(t)=\mathbf{X} \mathrm{e}^{\mathrm{i} \omega t}$, where $\mathrm{i}=\sqrt{-1}$. The PCE coefficients of $\mathbf{X}$ and $\mathbf{Y}$ satisfy

$$
\left(-\omega^{2} \widetilde{\mathbf{M}}+i \omega \widetilde{\mathbf{C}}+\widetilde{\mathbf{K}}\right) \mathbf{Y}(\omega)=\widetilde{\mathbf{F}_{\mathbf{0}}}
$$

The values of the physical parameters are given in Tables 1 and 2. Note that the deterministic quantities correspond to $\delta_{K}=0$.

\subsection{PC-Eigenvalues}

The eigenproblem to solve is

$$
\left(\widetilde{\mathbf{K}}_{0}+\widetilde{\mathbf{K}}_{1}-\widetilde{\lambda} \widetilde{\mathbf{M}}\right) \widetilde{\mathbf{V}}=\mathbf{0}
$$




$\begin{array}{ccc}\text { Eigenfrequencies } f(\mathrm{~Hz}) & 12.05 & 31.54 \\ \text { Modal damping ratio }(\%) & 0.25 & 0.66\end{array}$

Table 2: Modal characteristics of the deterministic system

The eigenvalues are the solution of

$$
\left|\widetilde{\mathbf{M}}^{-1}\left(\widetilde{\mathbf{K}}_{0}+\widetilde{\mathbf{K}}_{1}\right)-\widetilde{\lambda} \mathbf{I}\right|=0
$$

where $|\bullet|$ denotes the determinant of a matrix. Thus

$$
\left|\left(\mathbf{A}_{0} \otimes \mathbf{M}\right)^{-1}\left(\left(\mathbf{A}_{0}+\delta_{K} \mathbf{A}_{1}\right) \otimes \overline{\mathbf{K}}\right)-\tilde{\lambda} \mathbf{I}\right|=0
$$

The Kronecker product properties lead to the following problem

$$
\left|\mathbf{A}_{0}^{-1}\left(\mathbf{A}_{0}+\delta_{K} \mathbf{A}_{1}\right) \otimes\left(\mathbf{M}^{-1} \overline{\mathbf{K}}\right)-\tilde{\lambda} \mathbf{I}\right|=0
$$

Then

$$
\left|\left(\mathbf{I}+\delta_{K} \mathbf{A}_{0}^{-1} \mathbf{A}_{1}\right) \otimes\left(\mathbf{M}^{-1} \overline{\mathbf{K}}\right)-\tilde{\lambda} \mathbf{I}\right|=0
$$

and hence $\{\widetilde{\lambda}\}_{k}$ is a equal to $\left\{\lambda_{i} \times \omega_{j}^{2}\right\}_{(i, j)}$ where $\omega_{j}$ are the deterministic eigenfrequencies of the matrix pair $(\mathbf{K}, \mathbf{M})$, and $\lambda_{i}$ are the eigenvalues of $\left(\mathbf{I}+\delta_{K} \mathbf{A}_{0}^{-1} \mathbf{A}_{1}\right) . \lambda_{i}$ is a solution of the equation

$$
\left|\left(\mathbf{I}+\delta_{K} \mathbf{A}_{0}^{-1} \mathbf{A}_{1}\right)-\lambda \mathbf{I}\right|=0
$$

To simplify the problem, define $\alpha=(1-\lambda) / \delta_{K}$. From Eq. (23), $\alpha$ satisfies

$$
\left|\mathbf{A}_{0}^{-1} \mathbf{A}_{1}+\alpha \mathbf{I}\right|=0
$$


3.3. Case 1: Random variables $\xi$ follow a normal distribution

$\mathbf{A}_{0}$ and $\mathbf{A}_{1}$ are defined by

$$
\begin{aligned}
\mathbf{A}_{0}= & {\left[\begin{array}{cccc}
0 ! & 0 & \cdots & 0 \\
0 & \ddots & \cdots & \vdots \\
\vdots & \ddots & \ddots & \vdots \\
0 & \cdots & 0 & P !
\end{array}\right] } \\
\mathbf{A}_{1}= & {\left[\begin{array}{ccccc}
0 & 1 ! & 0 & \cdots & 0 \\
1 ! & 0 & 2 ! & \ddots & \vdots \\
0 & \ddots & \ddots & \ddots & 0 \\
\vdots & \ddots & \ddots & \ddots & P ! \\
0 & \cdots & 0 & P ! & 0
\end{array}\right] }
\end{aligned}
$$

and hence

$$
\mathbf{A}_{0}^{-1} \mathbf{A}_{1}=\left[\begin{array}{ccccc}
0 & 1 & 0 & \cdots & 0 \\
1 & 0 & 2 & \ddots & \vdots \\
0 & \ddots & \ddots & \ddots & 0 \\
\vdots & \ddots & \ddots & \ddots & P \\
0 & \cdots & 0 & 1 & 0
\end{array}\right]
$$

Then the problem to be solved is to find $\alpha$ such that

$$
D H_{P+1}(\alpha)=\left|\begin{array}{ccccc}
\alpha & 1 & 0 & \cdots & 0 \\
1 & \alpha & 2 & \ddots & \vdots \\
0 & \ddots & \ddots & \ddots & 0 \\
\vdots & \ddots & \ddots & \ddots & P \\
0 & \cdots & 0 & 1 & \alpha
\end{array}\right|=0
$$


Developing this determinant with respect to the last line, the following recursive equation is derived for $P \geq 2$

$$
D H_{P+1}(\alpha)=\alpha D_{P}(\alpha)-P D_{P-1}(\alpha)
$$

with

$$
\begin{aligned}
& D H_{2}(\alpha)=\alpha^{2}-1 \\
& D H_{1}(\alpha)=\alpha
\end{aligned}
$$

Hence $D H_{P+1}(\alpha)$ may be identified with $H_{P+1}(\alpha)$, the $(P+1)$ th Hermite polynomial, and the solution to Eq. (28) are the roots of $H_{P+1}$.

3.4. Case 2: Random variables $\xi$ follow a uniform distribution

$\mathbf{A}_{0}$ and $\mathbf{A}_{1}$ are defined by

$$
\begin{aligned}
\mathbf{A}_{0}= & {\left[\begin{array}{cccc}
1 & 0 & \cdots & 0 \\
0 & \ddots & \cdots & \vdots \\
\vdots & \ddots & \ddots & \vdots \\
0 & \cdots & 0 & \frac{1}{2 P+1}
\end{array}\right] } \\
\mathbf{A}_{1}= & \\
& {\left[\begin{array}{ccccc}
0 & 1 / 2 & 0 & \cdots & 0 \\
1 / 3 & 0 & 2 / 15 & \ddots & \vdots \\
0 & \ddots & \ddots & \ddots & 0 \\
\vdots & \ddots & \ddots & \ddots & \frac{P}{(2 P-1)(2 P+1)} \\
0 & \cdots & 0 & \frac{P}{(2 P-1)(2 P+1)} & 0
\end{array}\right] }
\end{aligned}
$$


Then the problem to be solved is to find $\alpha$ such that

$$
D L_{P+1}(\alpha)=\left|\begin{array}{ccccc}
\alpha & 1 / 3 & 0 & \cdots & 0 \\
1 & \alpha & 2 / 5 & \ddots & \vdots \\
0 & \ddots & \ddots & \ddots & 0 \\
\vdots & \ddots & \ddots & \ddots & \frac{P}{2 P+1} \\
0 & \cdots & 0 & \frac{P}{2 P-1} & \alpha
\end{array}\right|=0
$$

Developing this determinant with respect to the last line, the following recursive equation is derived for $P \geq 2$

$$
D L_{P+1}(\alpha)=\alpha D L_{P}(\alpha)-\frac{P}{2 P-1} \frac{P}{2 P+1} D L_{P-1}(\alpha)
$$

with

$$
\begin{aligned}
& D L_{2}(\alpha)=\alpha^{2}-\frac{1}{3} \\
& D L_{1}(\alpha)=\alpha
\end{aligned}
$$

This recursive equation shows $D_{P}$ belongs to a set of orthogonal polynomials. Further it may be showed that $D L_{P}$ is related to Legendre polynomial $L_{P}$ by

$$
D L_{P}(\alpha)=\frac{P !}{(2 P-1) ! !} L_{P}(\alpha)
$$

Then the solutions of Eq. (34) are the roots of $L_{P+1}$.

\section{Discussion}

[8] showed that the PCE coefficients given by Eq. (8) have PC-resonances that correspond to the spurious peaks depicted on the response moments. The last result shows that the PC-eigenfrequencies are equal to $\omega_{i} \times \sqrt{\left(1-\alpha_{j} \delta_{K}\right)}$, where the $\left\{\alpha_{j}\right\}$, for $0 \leq j \leq P$, are the roots of polynomials $H_{P+1}$ or 


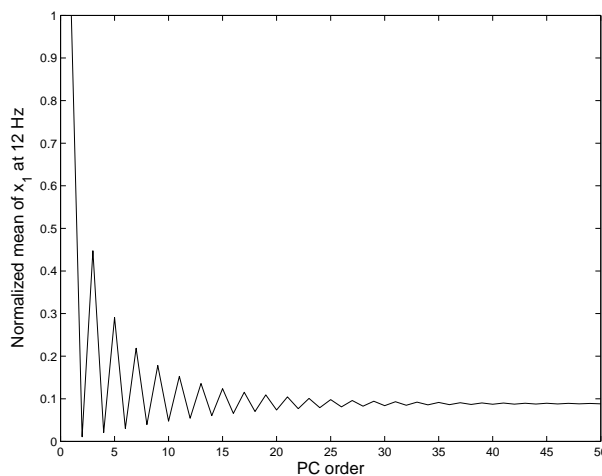

(a)

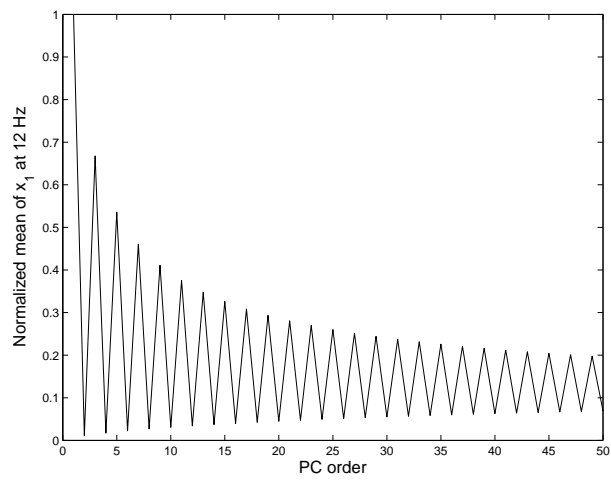

(b)

Figure 6: Evolution of the normalized mean response obtained at the first eigenfrequency $(12.05 \mathrm{~Hz})$ as a function of the order of the PCE: (a) Legendre (b) Hermite

$L_{P+1}$, depending of the nature of the random variable. As the roots of both Hermite and Legendre polynomials are symmetrical with respect to 0, PCeigenfrequencies are symmetrical about each deterministic eigenfrequency. Further, when $P$ is even, $\alpha=0$ is a root of $H_{P+1}$ or $L_{P+1}$, and so, in this case, the deterministic eigenfrequencies are in the set of the PC-eigenfrequencies.

To assess the dependence of the rate of convergence on the choice of polynomial type, the mean response is plotted as a function of the order of the PCE in Fig. 6. For a better comparison, the mean response is normalized so that the maximum is equal to unity and is obtained when the system is excited at $12.05 \mathrm{~Hz}$, which is the first deterministic eigenfrequency; Figs 1 and 4 show that the convergence was the slowest at this frequency. Fig. 6 shows that the convergence is much quicker with the Legendre polynomials.

The difference between Hermite and Legendre polynomial chaos is that in the first case the PC-eigenfrequencies spread from the deterministic eigenfrequencies whereas in the second case the $\mathrm{PC}$-eigenfrequencies remain in a 


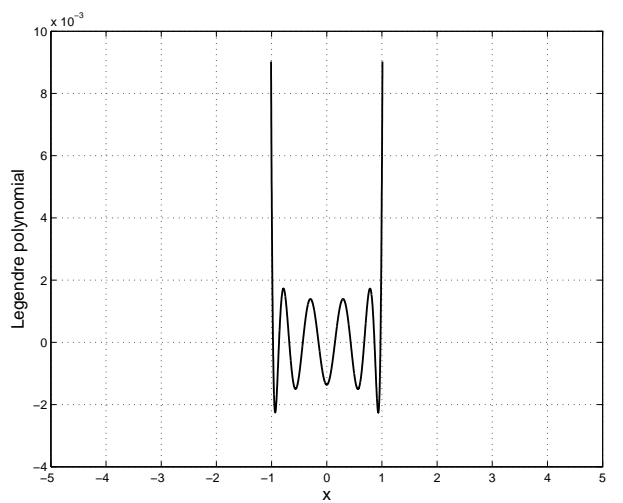

(a)

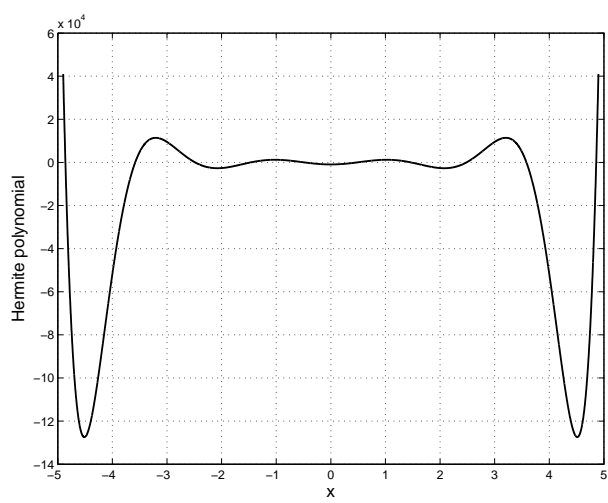

(b)

Figure 7: Polynomial chaos of order 10: (a) Legendre (b) Hermite

bounded interval close to the deterministic eigenfrequencies (see Figs. 7 and 8). So in the latter case, the PC eigenmodes will quickly smooth the response due to their closeness (see Fig. 9): in this case the PC-eigenmodes cannot be assumed as separate and then superimposed.

\subsection{PC-modal response}

According to Eq. (7), one has

$$
\mathbf{x}(\omega, \Xi)=\sum_{i=0}^{P} \mathbf{Y}_{i}(\omega) \Psi_{i}(\Xi)
$$

$\widetilde{\mathbf{Y}}$ is the response of a PC-dynamical system. Then, $\widetilde{\mathbf{Y}}$ may be expanded on the PC-eigenmodes as

$$
\tilde{\mathbf{Y}}(\omega)=\sum_{j=0}^{n(P+1)} q_{j}(\omega) \tilde{\mathbf{V}}_{j}
$$

where $\tilde{\mathbf{V}}_{j}$ is the $j$-th eigenvector of $(\widetilde{\mathbf{K}}, \widetilde{\mathbf{M}})$. Due to the definition of $\widetilde{\mathbf{K}}$ and $\widetilde{\mathbf{M}}, \tilde{\mathbf{V}}_{j}$ may be written as

$$
\tilde{\mathbf{V}}_{j}=\mathbf{W}_{k} \otimes \mathbf{V}_{m}
$$




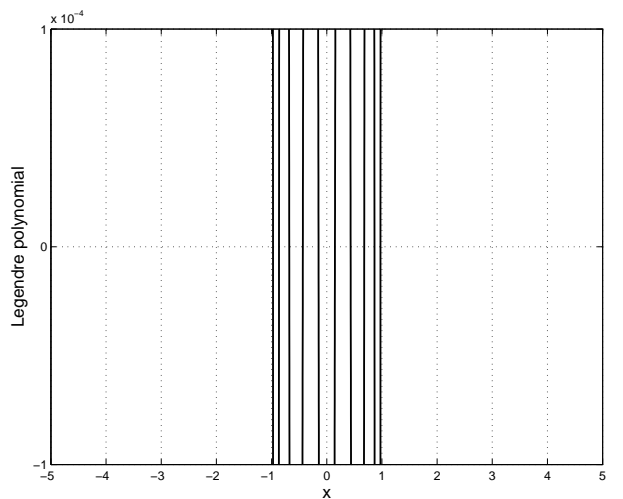

(a)

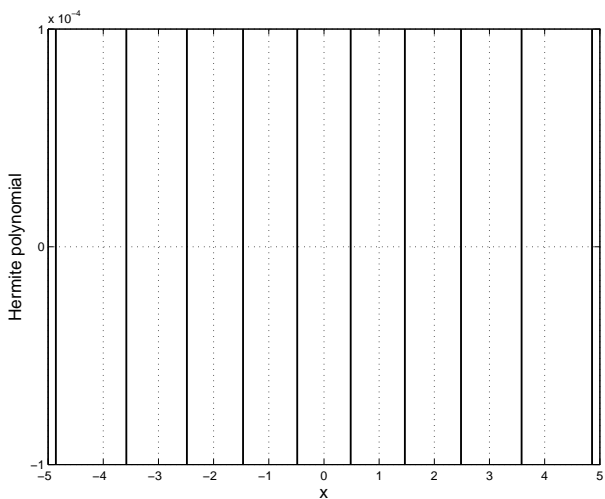

(b)

Figure 8: Roots of Polynomial chaos of order 10: (a) Legendre (b) Hermite

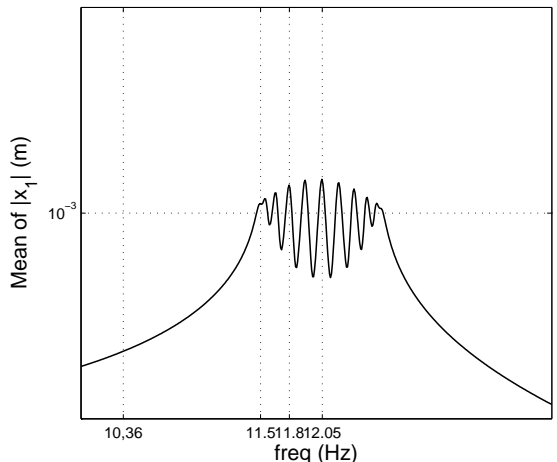

(a)

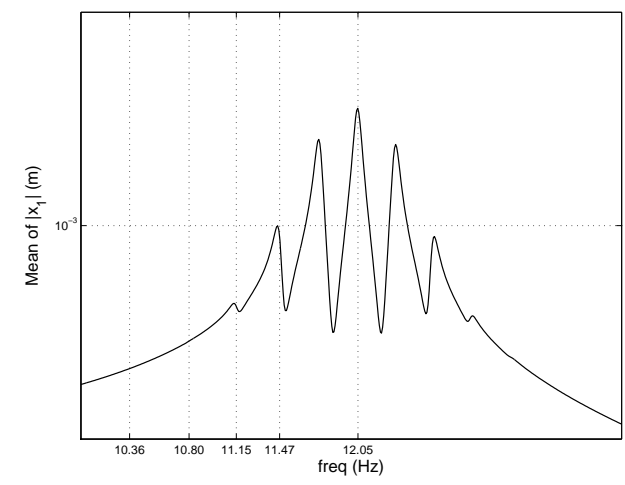

(b)

Figure 9: The mean response close to the first eigenfrequency: (a) Legendre (b) Hermite 
where $\mathbf{W}_{k}$ (resp. $\left.\mathbf{V}_{m}\right)$ is an eigenvector of $\left(\left(\mathbf{A}_{0}+\delta_{K} \mathbf{A}_{1}\right), \mathbf{A}_{0}\right)(\operatorname{resp} .(\mathbf{K}, \mathbf{M}))$, and $\tilde{\lambda}_{j}=\lambda_{k} \omega_{m}^{2}$. All of the modes are scaled with respect to the associated "mass" matrix.

Thus Eq. (40) may be rewritten as

$$
\tilde{\mathbf{Y}}(\omega)=\sum_{m=1}^{n} \sum_{k=0}^{P} q_{k m}(\omega) \mathbf{W}_{k} \otimes \mathbf{V}_{m}
$$

with

$$
q_{k m}(\omega)=\frac{\left(\mathbf{W}_{k} \otimes \mathbf{V}_{m}\right)^{t} \widetilde{\mathbf{F}}}{\lambda_{k} \omega_{m}^{2}-\omega^{2}+2 \mathrm{i} \tilde{\zeta}_{k m} \omega \omega_{m} \sqrt{\lambda_{k}}}
$$

Elements of $\widetilde{\mathbf{F}}$ are all equal to zero except $\widetilde{F}_{f}$, where $f$ is the dof excited by F (in the example presented below, $f=1$ ). Hence

$$
\left(\mathbf{W}_{k} \otimes \mathbf{V}_{m}\right)^{t} \widetilde{\mathbf{F}}=W_{k, 0} V_{m, f}
$$

From Eq. (42), each subvector $\mathbf{Y}_{i}$ of $\widetilde{\mathbf{Y}}$ is

$$
\mathbf{Y}_{i}=\sum_{m=1}^{n}\left(\sum_{k=0}^{P} q_{k m} W_{k, i}\right) \mathbf{V}_{m}
$$

Finally the response is

$$
\begin{aligned}
\mathbf{x} & =\sum_{m=1}^{n}\left(\sum_{i=0}^{P} \sum_{k=0}^{P} q_{k m}(\omega) W_{k, i} \Psi_{i}(\Xi)\right) \mathbf{V}_{m} \\
& =\sum_{m=1}^{n}\left(\sum_{i=0}^{P} \sum_{k=0}^{P} \frac{W_{k, 0} W_{k, i} \Psi_{i}(\Xi)}{\lambda_{k} \omega_{m}^{2}-\omega^{2}+2 \mathrm{i} \tilde{\zeta}_{k m} \omega \omega_{m} \sqrt{\lambda_{k}}}\right) V_{m, f} \mathbf{V}_{m}
\end{aligned}
$$

Then the modal factor of dof $r$ is

$$
H_{k m, r}=W_{k, 0}\left(\sum_{i=0}^{P} W_{k, i} \Psi_{i}(\Xi)\right) V_{m, f} V_{m, r}
$$




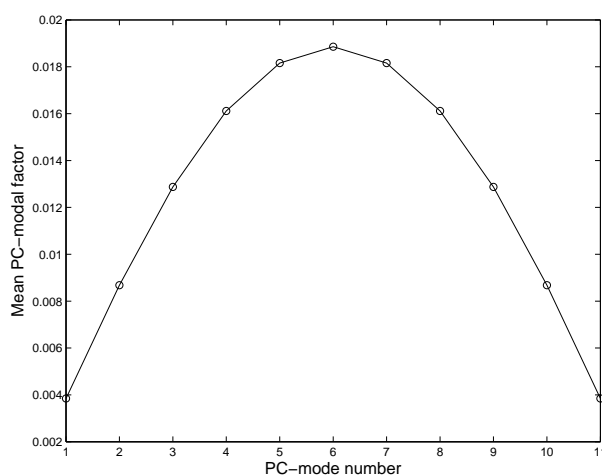

(a)

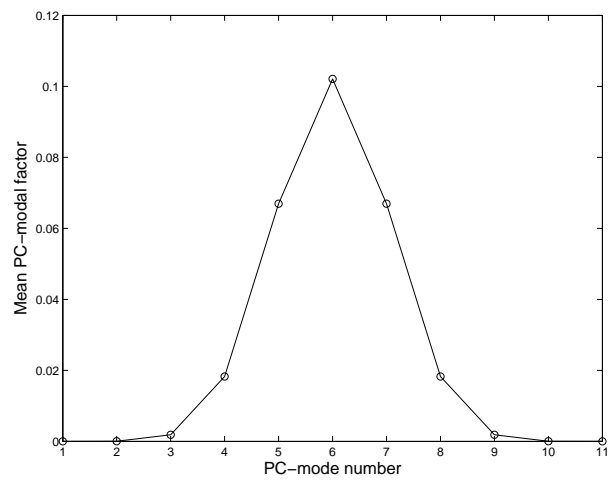

(b)

Figure 10: Modal factors, for $P=10$ : (a) Legendre (b) Hermite

$H_{k m, r}$ is then a random variable. The mean of this modal factor, $\bar{H}_{k m, r}$, is also the modal factor of the mean of $\mathbf{x}$

$$
\bar{H}_{k m, r}=W_{k, 0}^{2} V_{m, f} V_{m, r}
$$

The mean modal factors of dof $r$ are plotted in Fig. 10 for both the Legendre and Hermite cases. As only the first 11 mean modal factors are plotted, they are all associated with the first deterministic eigenfrequency (indeed, $P=10$ ). This shows that the Hermite case tends to weight more highly the PC-modes around the deterministic eigenfrequency, which tends to diminish the smoothing effect due to the superimposed PC-modes. Although this feature exists for the Legendre case, it is not as strong.

\section{Conclusion}

Dynamic response analysis in the frequency-domain using polynomial chaos (PC) expansion is considered in this note. Particular attention is placed on the response statistics around the deterministic resonance frequencies, 
where the convergence of the PC solution is known to be more erroneous than at other frequency values. The fundamental reason behind this phenomenon lies in the nature of the roots of the polynomials used for the PCE. The PC-eigenfrequencies are related to the roots of the PC used to derive the expansion. In the case of Hermite polynomials, the PC-eigenfrequencies spread from the deterministic eigenfrequencies, which leads to a slow convergence. In contrast, for Legendre polynomials the PC eigenfrequencies remain in a closed interval. Consequently the PC-eigenmodes are not separated and they are superimposed, which in turn leads to a smoothing effect and to a quicker convergence. The theoretical idea proposed here can be extended to other polynomials, which would be necessary if the underlying probability density function of the random variables was different to that considered here.

\section{Acknowledgments}

J-J. Sinou acknowledges the support of the Institut Universitaire de France.

\section{APPENDIX: Uncertain mass and stiffness matrices}

To derive closed-form expressions, the uncertain mass and stiffness matrices are assumed to be of the form

$$
\begin{aligned}
\mathbf{M} & =\overline{\mathbf{M}}\left(1+\delta_{M} \xi\right) \\
\mathbf{K} & =\overline{\mathbf{K}}\left(1+\delta_{K} \xi\right)
\end{aligned}
$$

where $\xi$ is a random variable.

The corresponding eigenproblem is then

$$
\left(\widetilde{\mathbf{K}}_{0}+\widetilde{\mathbf{K}}_{1}-\widetilde{\lambda}\left(\widetilde{\mathbf{M}}_{0}+\widetilde{\mathbf{M}}_{1}\right)\right) \widetilde{\mathbf{V}}=\mathbf{0}
$$


The eigenvalues are the solution of

$$
\left|\left(\widetilde{\mathbf{M}}_{0}+\widetilde{\mathbf{M}}_{1}\right)^{-1}\left(\widetilde{\mathbf{K}}_{0}+\widetilde{\mathbf{K}}_{1}\right)-\widetilde{\lambda} \mathbf{I}\right|=0
$$

Thus

$$
\left|\left(\left(\mathbf{A}_{0}+\delta_{M} \mathbf{A}_{1}\right) \otimes \overline{\mathbf{M}}\right)^{-1}\left(\left(\mathbf{A}_{0}+\delta_{K} \mathbf{A}_{1}\right) \otimes \overline{\mathbf{K}}\right)-\tilde{\lambda} \mathbf{I}\right|=0
$$

The Kronecker product properties lead to the following problem

$$
\left|\left(\mathbf{A}_{0}+\delta_{M} \mathbf{A}_{1}\right)^{-1}\left(\mathbf{A}_{0}+\delta_{K} \mathbf{A}_{1}\right) \otimes\left(\overline{\mathbf{M}}^{-1} \overline{\mathbf{K}}\right)-\widetilde{\lambda} \mathbf{I}\right|=0
$$

$\delta_{M}$ is now assumed to be a very small parameter, so that to first order in $\delta_{M}$

$$
\left(\mathbf{A}_{0}+\delta_{M} \mathbf{A}_{1}\right)^{-1} \simeq \mathbf{A}_{0}^{-1}-\delta_{M} \mathbf{A}_{0}^{-1} \mathbf{A}_{1} \mathbf{A}_{0}^{-1}
$$

Then

$$
\left(\mathbf{A}_{0}+\delta_{M} \mathbf{A}_{1}\right)^{-1}\left(\mathbf{A}_{0}+\delta_{K} \mathbf{A}_{1}\right) \simeq \mathbf{I}+\left(\delta_{K}-\delta_{M}\right) \mathbf{A}_{0}^{-1} \mathbf{A}_{1}
$$

and

$$
\left|\left(\mathbf{I}+\left(\delta_{K}-\delta_{M}\right) \mathbf{A}_{0}^{-1} \mathbf{A}_{1}\right) \otimes\left(\overline{\mathbf{M}}^{-1} \overline{\mathbf{K}}\right)-\tilde{\lambda} \mathbf{I}\right|=0
$$

Hence, the equivalent to Eq. (23) is obtained by replacing $\delta_{K}$ by $\left(\delta_{K}-\delta_{M}\right)$. As a consequence, the same conclusions can be drawn with uncertain mass and stiffness matrices provided $\delta_{M}$ is small enough to make Eq. (.7) valid.

[1] Blatman, G. and Sudret, B. (2010). "An adaptive algorithm to build up sparse polynomial chaos expansions for stochastic finite element analysis." Probabilistic Engineering Mechanics, 25(2), 183 - 197.

[2] Eldred, M. S. and Burkardt, J. (2009). "Comparison of non-intrusive polynomial chaos and stochastic collocation methods for uncertainty quantification." Vol. 2009-0976, 47th AIAA Aerospace Sciences Meeting. 
[3] Falsone, G. and Ferro, G. (2005). "A method for the dynamical analysis of fe discretized uncertain structures in the frequency domain." Computer Methods in Applied Mechanics and Engineering, 194, 4544-4564.

[4] Falsone, G. and Impollonia, N. (2002). "A new approach for the stochastic analysis of finite element modelled structures with uncertain parameters." Computer Methods in Applied Mechanics and Engineering, 191, 5067-5085.

[5] Ghanem, R. G. and Ghosh, D. (2007). "Efficient characterization of the random eigenvalue problem in a polynomial chaos decomposition." International Journal for Numerical Methods in Engineering, 72, 486-504.

[6] Ghanem, R. G. and Spanos, P. D. (1991). Stochastic Finite Elements: A Spectral Approach. Springer-Verlag, New York, USA.

[7] Jacquelin, E., Adhikari, S., Sinou, J.-J., and Friswell, M. (2015a). "Polynomial chaos expansion in structural dynamics: Accelerating the convergence of the first two statistical moment sequences." Journal of Sound and Vibration, 356, 144154.

[8] Jacquelin, E., Adhikari, S., Sinou, J.-J., and Friswell, M. I. (2015b). "The polynomial chaos expansion and the steady-state response of a class of random dynamic systems." ASCE Journal of Engineering Mechanics, 141(4), 04014145.

[9] Kundu, A. and Adhikari, S. (2014). "Transient response of structural dynamic systems with parametric uncertainty." ASCE Journal of Engineering Mechanics, 140(2), 315-331. 
[10] Kundu, A. and Adhikari, S. (2015). "Dynamic analysis of stochastic structural systems using frequency adaptive spectral functions." Probabilistic Engineering Mechanics, 39(1), 23-38.

[11] Sepahvand, K., Marburg, S., and Hardtke, H.-J. (2010). "Uncertainty quantification in stochastic systems using polynomial chaos expansion." International Journal of Applied Mechanics, 2(2), 305-353.

[12] Settineri, D. and Falsone, G. (2014). "An APDM-based method for the analysis of systems with uncertainties." Computer Methods in Applied Mechanics and Engineering, 278, 828-852.

[13] Sinou, J.-J. and Jacquelin, E. (2015). "Influence of polynomial chaos expansion order on uncertain rotor system response." Mechanical System and Signal Processing, 50-51, 718-731.

[14] Verhoosel, C. V., Gutiérrez, M. A., and Hulshoff, S. J. (2006). "Iterative solution of the random eigenvalue problem with application to spectral stochastic finite element systems." International Journal for Numerical Methods in Engineering, 68(4), 401-424. 